\title{
ORIGINAL
}

\section{ACCIDENTES ATENDIDOS EN UN ÁREA BÁSICA DE SALUD DE GIRONA, ESPAÑ}

Elena Soriano Suárez, Jesús Sánchez Rodríguez, Anna Oliva Arbat, José Cristóbal Buñuel Álvarez, Remei Girona Bastús y Concepció Morera Jordán.

Área Básica de Salud Girona-4. Institut Català de la Salut. Girona

\section{RESUMEN}

Fundamentos: Los accidentes constituyen una patología poco estudiada en el ámbito de la Atención Primaria. Son una de las consultas más frecuentes en los servicios de urgencias y los Centros de Atención Primaria realizan la primera asistencia a la mayoría de los accidentados. Conocer la incidencia y las características clínico-epidemiológicas de los accidentes atendidos en una Área Básica de Salud puede aportar información sobre cuáles pueden ser susceptibles de actividades de prevención.

Métodos: Diseño: estudio descriptivo. Emplazamiento: atención primaria. Muestra: todos los pacientes (389) que fueron atendidos por accidente en el Centro de Atención Primaria, entre octubre-98 y mayo-99. Variables: edad, sexo, lugar del accidente, tipo de lesión, localización, agentes implicados, intencionalidad, pruebas complementarias, tratamiento y derivación. Análisis estadísticos: estimación de medias, desviación estándar, estimación de proporciones e intervalos de confianza del 95\%.

Resultados: Incidencia: 4,1\% (IC $95 \%: 3,7-4,5 \%)$. Sexo: varones $59 \%$ (IC $95 \%: 54,2-64 \%$ ) y mujeres $40,9 \%$ (IC $95 \%$ : $36-45,8 \%$ ). Edad: menores de 20 años, el $50,4 \%$ (IC $95 \%: 45,4-55,4 \%) ;$.Actividad de mayor accidentalidad: ocio $24,4 \%$ (IC $95 \%$ : 20,2-28,7\%). Lugar: hogar 36,2\% (IC $95 \%$ : $31,5-41 \%)$. Lesión más frecuente: contusiones $39,6 \%$ (IC95\%:34,7-44,4\%).Localización más frecuente: extremidad superior 37,5\% (IC $95 \%$ : 32,7-42,3\%); Agente mayoritariamente implicado: herramientas y máquinas: $15,9 \%$ (IC $95 \%: 12,3-19,6 \%$ ). El 92,2\% (IC95\%: 89,3-94,7\%) fueron casuales. Tipo de visita: el 83,3\% (IC95\%: 79,6-87\%) fueron atendidos con carácter urgente; el 79,5\% (IC95\%:75,4-83,5\%) recibió tratamiento con cura y/o fármacos. El $9,8 \%$ (IC $95 \%: 6,8-12,7 \%$ ) requirió derivación hospitalaria, Un 13,3\% (IC $95 \%$ : 0-16,7\%) requirió pruebas complementarias.

Conclusiones: El mayor porcentaje de accidentalidad se da en población joven, por lo que se evidencia la necesidad de incorporar intervenciones de educación sanitaria para la prevención de accidentes dirigidas a dicha población.

Palabras clave: Accidentes. Atención Primaria. Adolescen-

Correspondencia:

Elena Soriano Suárez

C/ Nuestra Sra. De Montserrat n. ${ }^{\circ} 2,5 .^{\circ}-4 .^{\text {a }}$

Figueres

17600 Girona

\section{ABSTRACT}

\section{Accidents Attended in a Basic Health Area of Girona, Spain}

Background: Accidents have been largely unstudied in the area of Primary Care. They are one of the most frequent motives for consultation in the Emergency Services and the first assistance that accident victims receive is usually in primary care centres. Establishment of the incidence and clinicoepidemiological characteristics of the accidents attended in a Basic Health Area can provide important information about which of these could be susceptible to preventive actions.

Methods: Design: descriptive study. Location: primary care: Sample: all the patients attended for accidents (389) in the Primary Care Centre between October 1998 and May 1999. Variables: age, sex, place of the accident, type of lesion, location of lesions, agents involved, intentionality, complementary tests, treatment and referral. Statistical analysis: estimation of means, standard deviation, proportions and $95 \%$ confidence intervals.

Results: Incidence: 4.1\% (CI95\%: 3.7-4.5\%). Sex: males $59 \%$ (CI95\% $54.2-64 \%$ ) and females $40.9 \%$ (CI $95 \%: 36-45.8 \%)$ Age: younger than 20 years, $50.4 \%\left(\mathrm{CI}_{95 \%}\right.$ : 45.4-55.4\%). Most common activity associated with accidents: leisure $24.4 \%$ (CI ${ }_{95 \%}$ : 20.2-28.7\%). Place: home 36.2\% (C95\%: 31.5-41\%). Most frequent lesion: contusion 39.6\% (CI $95 \%$ : 34.7-44.4\%). Most frequent site of lesion: arms 37.5\% (CI95\%: 32.7-42.3\%). Most common agent involved: tools and machinery $15.9 \%$ (CI $\left.{ }_{95 \%}: 12.3-19.6 \%\right)$. Of these, $92.2 \%\left(\mathrm{CI}_{95 \%}: 89.3-94.7 \%\right)$ were accidental. Type of visit: $83.3 \%\left(\mathrm{CI}_{95 \%}\right.$ : $\left.79.6-87 \%\right)$ were attended as emergencies; $79.5 \%$ (CI95\%: 75.4-83.5\%) received treatment with dressings and/or medication. Of these, $9.8 \%$ ( $\mathrm{CI}_{95 \%}$ : $6.8-12.7 \%$ ) required referral to a hospital, $13.3 \%$ (CI $95 \%$ : 10-16.7\%) required complementary tests.

Conclusions: Most accidents occur in young people and educational campaigns to prevent accidents and directed towards this population group are clearly needed.

Key words: Accidents. Primary care. Adolescent. 


\section{INTRODUCCIÓN}

Los accidentes son uno de los motivos de consulta más frecuentes de los servicios de urgencias, tanto en el ámbito hospitalario como extrahospitalario ${ }^{1-6}$. Los Centros de Atención Primaria (CAP) desempeñan un papel importante en la primera asistencia de las personas accidentadas. La mayoría de los accidentes no suelen poner en peligro la vida. Sin embargo, deben ser tenidos en cuenta por su alta prevalencia y por ser susceptibles de actividades de prevención.

Numerosos estudios del ámbito hospitalario han analizado este tema. Muchos sugieren que entre una y dos terceras partes de las personas atendidas por accidente podrían haber sido tratadas en un $\mathrm{CAP}^{7-13}$. Este fenómeno también está descrito en otros paí ses $^{14-19}$. Los sistemas de información existentes nos facilitan datos sobre la atención en urgencias pero no sobre la accidentabilidad atendida por la medicina de familia.

Por otra parte, los accidentes constituyen una patología poco estudiada en el ámbito de la AP. Exceptuando los trabajos elaborados en la edad pediátrica, los estudios descriptivos sobre accidentes en este ámbito son escasos ${ }^{6}$. Y, sin embargo, es importante conocerla y estudiar su evolución para instaurar intervenciones en los grupos de riesgo y las situaciones susceptibles de ser sensibles a ellas.

El presente trabajo realiza un seguimiento completo de los accidentes como motivo de consulta en AP a lo largo de las 24 horas del día y en todo tipo de consulta (cita previa, programada, atención continuada, urgente y domiciliaria), en contraste con otro estudio que recoge la actividad atendida sólo en un servicio de urgencias extrahospitalarias ${ }^{6}$.

El objetivo de este trabajo es, pues, conocer la incidencia acumulada y las características clínico-epidemiológicas de los accidentes asistidos en primera instancia en el Área Básica de Salud (ABS) de Girona 4, la cual atiende a un total de 9.442 habitantes, y abarca los municipios de St. Gregori, Canet
d'Adri, St. Martí de Llémena y una parte del término municipal de Girona ${ }^{20}$. Se trata de una población joven (el 53\% tienen entre 15 y 44 años) y que utiliza de forma preferente los servicios del ABS, la cual pertenece al modelo reformado de Asistencia Primaria (AP).

\section{MATERIAL Y MÉTODOS}

Se realizó un estudio descriptivo longitudinal con muestreo consecutivo, durante el periodo comprendido entre octubre de 1998 y mayo de 1999, recogiéndose en una hoja de registro diseñada para tal fin todos los accidentes atendidos en el ABS durante las 24 horas del día, en cualquier tipo de consulta (espontánea, programada, urgencias, atención continuada y domiciliaria).

Se excluyó a aquellos individuos que ya habían sido atendidos previamente por el mismo accidente en otros centros (evitando así las atenciones duplicadas con otros ámbitos asistenciales) y a los pacientes que no residían dentro de los límites del ABS, para conocer exactamente la accidentabilidad de la población de referencia atendida de forma inicial por el CAP.

Se definió el término accidente, siguiendo los criterios de la $\mathrm{WONCA}^{21}$, como aquella situación súbita, inesperada y fortuita de la que se deriva una lesión en un individuo. En la hoja de registro se detallaron:

1. Datos personales: edad y sexo.

2. Datos relativos a la asistencia sanitaria: fecha y hora; tipo de consulta (espontánea, programada, atención continuada, urgencias, domiciliaria); tipo de lesión (contusión, torsión o esguince, fracturas, traumatismo craneoencefálico, herida abierta, erosión o rascada, quemadura, mordedura, picadura de insecto, contracturas, cuerpos extraños, intoxicaciones, amputaciones); localización anatómica de la lesión (mano, brazo, pierna, pie, hemitronco superior o inferior, cuero cabelludo, ojo, nariz, orejas, boca, piel facial, cuello); tratamiento: reposo, curas (entendiendo como tal: cura tópi- 
ca, vendaje, inmovilización, sutura, extracción de cuerpo extraño y oclusión ocular) y fármacos; pruebas complementarias solicitadas; derivación del paciente a otros servicios (servicio hospitalario de urgencias, oftalmología, otorrinolaringología y traumatología).

3. Datos relativos a la situación en la que sucedió el accidente: fecha y hora; lugar (casa, aire libre, escuela, trabajo); actividad (ir en vehículo, trabajar, bajar o subir escaleras, actividad de ocio o juego, deportes, agresión, actividad doméstica, caminar o correr); agentes implicados (se agruparon en vehículos/herramientas/maquinas; animales/personas, arboles/plantas; líquidos/tóxicos/explosivos; suelo/aceras/desniveles); presencia de tóxicos o fármacos relacionados; intencionalidad (diferenciando entre autoagresión, heteroagresión y accidente casual).

\section{Análisis estadístico}

Cálculo del tamaño de la muestra: se estimó que era necesaria una muestra de 384 personas para una proporción esperada de 0,5 , un error alfa $=0,05$ y una precisión de 0,05 . Finalmente participaron 389 individuos. Estadística: medidas de tendencia central: medias y proporciones; medidas de dispersión: desviación estándar (DE). Las estimaciones se acompañan de su correspondiente intervalo de confianza del $95 \%$ $\left(\mathrm{IC}_{95 \%}\right)$. Se utilizó la prueba de $\mathrm{chi}^{2}$ para la comparación de las variables cualitativas.

\section{RESULTADOS}

La incidencia acumulada de los accidentes fue el $4,1 \%$ de todas las consultas efectuadas en nuestro centro de salud durante el periodo de estudio ( $\mathrm{IC}_{95 \%}: 3,7-4,5 \%$ ). El $50,4 \%\left(\mathrm{IC}_{95 \%}: 45,4-55,4 \%\right)$ se produjeron en personas menores de 20 años, seguido en frecuencia por los individuos de entre $21 \mathrm{y}$ 30 años $(16,2 \%)\left(\mathrm{IC}_{95 \%}: 10,2-17 \%\right)$. Las personas mayores de 70 años fueron las que menos consultaron por este motivo: $5,2 \%$ $\left(\mathrm{IC}_{95 \%}: 2,9-7,3 \%\right.$ ) (figura 1 ).

Figura 1

Distribución de los accidentes por edad y sexo

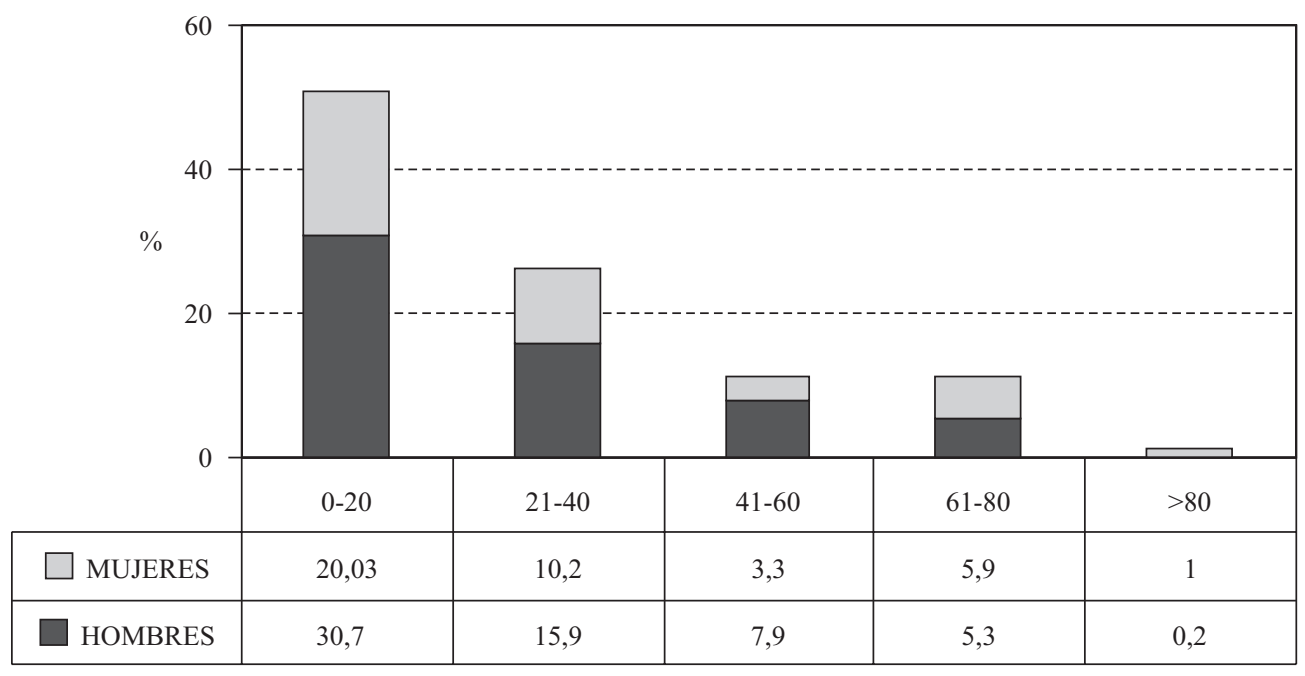

Años 
Los varones se accidentaron más frecuentemente que las mujeres, dando lugar al $59,1 \%$ de las consultas $(\mathrm{p}<0,05)$. La media de edad de los varones fue de 25,4 años $(\mathrm{DE}=20,2)\left(\mathrm{IC}_{95 \%}: 22,7-27,9\right.$ años$)$ y la de las mujeres 28,65 años $(\mathrm{DE}=23,1)\left(\mathrm{IC}_{95 \%}\right.$ : 25,1-32,2 años). El rango de edad estuvo comprendido entre los 3 meses y los 95 años (figura 1).

Las actividades en las que se produjeron accidentes están representadas en la tabla 1 .

Tabla 1

Clasificación de los accidentes según la actividad realizada

\begin{tabular}{|lcc|}
\hline Actividad & Porcentaje & IC 95\%* \\
\hline Jugar/Act. de ocio & 24,4 & $20,2-28,7 \%$ \\
Act. Doméstica & 17,5 & $13,7-21,3 \%$ \\
Caminar/Correr & 13,4 & $10-16,7 \%$ \\
Trabajar & 10,5 & $7,5-13,6 \%$ \\
Deporte & 10,0 & $7-13 \%$ \\
Vehículo & 8,3 & $5,5-11 \%$ \\
Bajar/Subir escaleras & 5,1 & $2,9-7,3 \%$ \\
Pelea/Agresión & 2,3 & $0,8-3,8 \%$ \\
Otros & 5,1 & $2,9-7,3 \%$ \\
No consta & 3,3 & $1,6-5,1 \%$ \\
\hline
\end{tabular}

* IC95\%: Intervalo de confianza del $95 \%$.

Las más frecuentes fueron las relacionadas con el ocio, con un $24,4 \%(p<0,05)$; éstas fueron más frecuentes en hombres $(53,7 \%)$. La mayoría de personas que tuvieron accidentes jugando o realizando actividades de ocio tenían entre 0 y 10 años $(56,8 \%)$ $\left(\mathrm{IC}_{95 \%}: 46,9-66,8 \%\right)$. La segunda actividad más frecuente fue la doméstica $(17,5 \%)$ $\left(\mathrm{IC}_{95 \%}: 13,7-21,3 \%\right)$, sin existir en esta categoría diferencias estadísticamente significativas entre hombres y mujeres; esta causa fue la más frecuente en las edades comprendidas entre 20 y 30 años. Las personas mayores de 70 años se accidentaron más frecuentemente mientras caminaban (caídas con o sin elemento precipitante) ${ }^{29}$.

El lugar donde se produjo el accidente se muestra en la tabla 2. Destaca que en los
Tabla 2

Clasificación de los accidentes según el lugar donde se produjeron

\begin{tabular}{|lcc|}
\hline Lugar accidente & Porcentaje & IC 95\%* \\
\hline Casa & 36,2 & $31,5-41 \%$ \\
Aire libre & 35,2 & $30,5-40 \%$ \\
Escuela & 14,9 & $11,4-18,4 \%$ \\
Trabajo & 9,5 & $6,6-12,4 \%$ \\
Otros & 3,3 & $1,6-5,1 \%$ \\
No consta & 0,8 & $-0,1-1,6 \%$ \\
\hline
\end{tabular}

* IC95\%: Intervalo de confianza del 95\%

accidentes laborales existe una relación de hombres respecto a las mujeres de $3: 1$. Los niños menores de 10 años tuvieron más accidentes en su domicilio (41,1\%) ( $\mathrm{IC}_{95 \%}$ : $33-49,3 \%)$ que en la escuela $(35,8 \%)$ $\left(\mathrm{IC}_{95 \%}: 26,1-45,4 \%\right)$. De todas las personas que tuvieron un accidente al aire libre, el $52,6 \%\left(\mathrm{IC}_{95 \%}: 44,2-60,9 \%\right)$ fueron jóvenes de edades comprendidas entre 11 y 30 años.

El tipo de lesión sufrida en el accidente se refiere en la tabla 3. La situación anatómica de las lesiones se refiere en la tabla 4. Los

Tabla 3

Clasificación de los accidentes según el tipo de lesión que produjeron

\begin{tabular}{|lcc|}
\hline \multicolumn{1}{|c}{ Tipo de lesión } & Porcentaje & IC 95\%* \\
\hline Contusión & 39,6 & $34,7-44,4 \%$ \\
Herida abierta & 21,1 & $17-25,1 \%$ \\
Torsión o esguince & 11,8 & $8,6-15 \%$ \\
Erosión o rascada & 8,5 & $5,7-11,3 \%$ \\
Cuerpo extraño & 6,4 & $4-8,9 \%$ \\
Quemadura & 3,6 & $1,7-5,4 \%$ \\
Mordedura & 2,6 & $1-4,1 \%$ \\
Picadura insecto & 2,3 & $0,8-3,8 \%$ \\
Contractura & 2,1 & $0,6-3,5 \%$ \\
Fractura & 0,8 & $-0,1-1,6 \%$ \\
Intoxicación & 0,3 & $-0,2-0,8 \%$ \\
TCE ** & 0,3 & $-0,2-0,8 \%$ \\
Otros & 0,8 & $-0,1-1,6 \%$ \\
\hline
\end{tabular}

* IC95\%: Intervalo de confianza del 95\%; ** TCE: Traumatismo craneoencefálico. 
Tabla 4

Clasificación de los accidentes según el lugar anatómico afectado

\begin{tabular}{|lcc|}
\hline \multicolumn{1}{|c}{ Lugar anatómico } & Porcentaje & IC 95\%* \\
\hline Mano & 29,3 & $24,8-33,8 \%$ \\
Pie & 15,2 & $11,6-18,7 \%$ \\
Pierna & 11,6 & $8,4-14,7 \%$ \\
Brazo & 8,2 & $5,5-11 \%$ \\
Piel facial & 8,2 & $5,5-11 \%$ \\
Ojo & 6,7 & $4,2-9,2 \%$ \\
Cuero cabelludo & 3,9 & $1,9-5,8 \%$ \\
Cuello & 3,1 & $1,4-4,8 \%$ \\
Boca & 2,8 & $1,2-4,5 \%$ \\
Hemitronco inferior & 2,6 & $1-4,1 \%$ \\
Hemitronco superior & 2,3 & $0,8-3,8 \%$ \\
Nariz & 0,8 & $-0,1-1,6 \%$ \\
Oreja & 0,5 & $-0,2-1,2 \%$ \\
Más de 1 localización & 4,6 & $2,5-6,7 \%$ \\
Otros & 0,3 & $-0,2-0,8 \%$ \\
\hline
\end{tabular}

* IC95\%: Intervalo de confianza del 95\%.

agentes materiales implicados en la producción de accidentes fueron: vehículos, herramientas y máquinas en el $15,9 \%\left(\mathrm{IC}_{95 \%}\right.$ : 12,3-19,6), caídas al suelo en el mismo nivelen el $14,4 \%\left(\mathrm{IC}_{95 \%}: 10,9-17,9 \%\right)$, contu- sión con animales o personas en el $13,4 \%$ $\left(\mathrm{IC}_{95 \%}: 10-6,7 \%\right)$. En tres casos hubo un tóxico implicado. De los individuos que sufrieron accidentes con vehículos, herramientas o máquinas, el 67,7\% fueron hombres $(p<0,05)$. No hubo diferencias significativas entre los días de la semana, aunque la asistencia fue menor en sábados y domingos $(\mathrm{p}>0,05)$. El 92,2\% ( $\left.\mathrm{IC}_{95 \%}: 89,3-94,7\right)$ fueron accidentes casuales. $\mathrm{El} \mathrm{4,1 \%}\left(\mathrm{IC}_{95 \%}\right.$ : 2,1-6,1\%) de las consultas correspondió a heteroagresiones. No se detectó ningún caso de autoagresión. El 83,3\% ( $\mathrm{IC}_{95 \%}$ : $79,6-87 \%$ ) de los accidentes fueron atendidos sin haber solicitado cita previa. A ésta acudieron un 10,8\% ( $\left.\mathrm{IC}_{95 \%}: 7,7-13,9 \%\right)$ de pacientes. Dos personas requirieron atención en su domicilio.

En cuanto al tratamiento, el 58,4\% $\left(\mathrm{IC}_{95 \%}\right.$ : $53,5-63,3 \%)$ de los casos precisó cura. Al $11,6 \%\left(\mathrm{IC}_{95 \%}: 8,4-14,7 \%\right)$ se le prescribió únicamente terapia farmacológica. El 9,5\% $\left(\mathrm{IC}_{95 \%}: 6,6-12,4 \%\right)$ requirió ambos tratamientos (figura 2). Un 13,3\% ( $\mathrm{IC}_{95 \% \text { : }}$ $10-6,7 \%$ ) requirió la realización de pruebas complementarias, de las cuales el 12,3\% $\left(\mathrm{IC}_{95 \%}: 9,1-15,6 \%\right)$ fueron radiografías; el

Figura 2

Medidas terapéuticas utilizadas en los accidentes atendidos en el Área Básica de Salud.

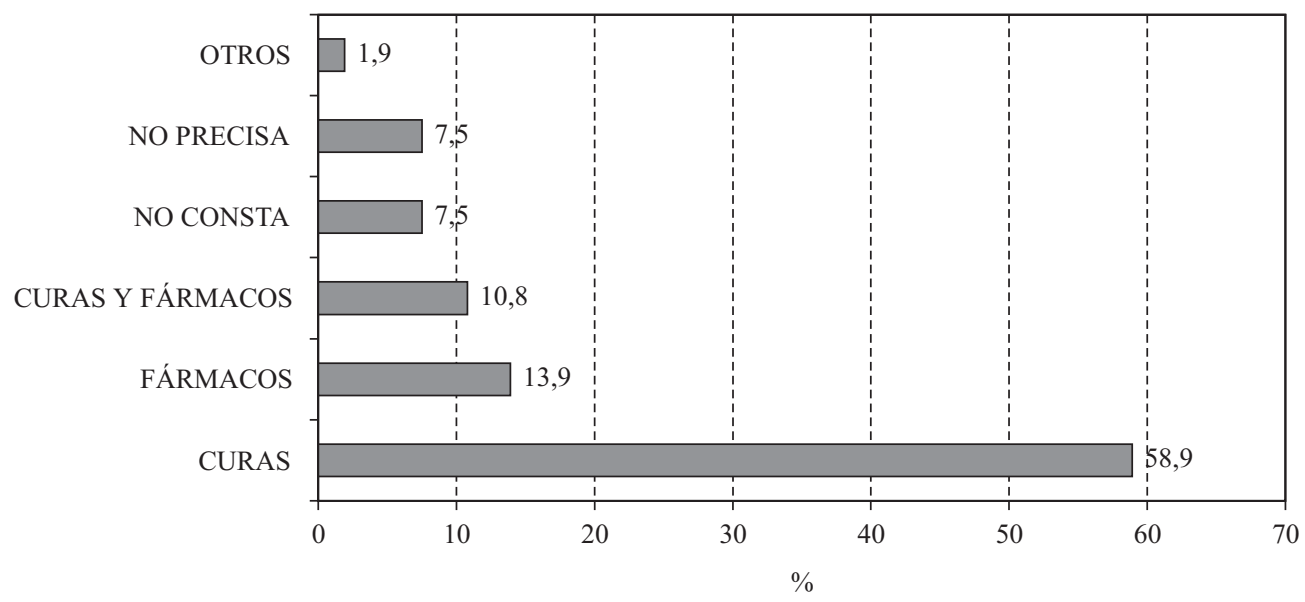


$1 \%\left(\mathrm{IC}_{95 \%}:-0,2-2\right)$ precisó tira reactiva de orina. El 9,8\% ( $\left.\mathrm{IC}_{95 \%}: 6,8-12,7 \%\right)$ de los pacientes fueron remitidos al servicio de urgencias hospitalario. Un 1,5\% ( $\mathrm{IC}_{95 \%}$ : $0,3-2,8 \%$ ) de los pacientes fueron derivados a otros especialistas para ser controlados conjuntamente: uno fue derivado al odontólogo, tres al oftalmólogo y dos al traumatólogo.

\section{DISCUSIÓN}

La incidencia de accidentes obtenida en el presente estudio, $4,1 \%$, es ligeramente inferior a la publicada por Aarseth et al, que en un trabajo similar constataron que un $9,1 \%$ de la población acudía a su médico de cabecera a causa de un accidente ${ }^{22}$.

Si comparamos los resultados con otro estudio semejante realizado en nuestro país ${ }^{6} \mathrm{se}$ constata que, en ambos, los accidentes de tráfico $(10,5 \%)$ y de trabajo incluidos los accidentes in itinere $(8,3 \%)$ representan un porcentaje algo más bajo (18,8\%), mientras que los accidentes domésticos y en actividades de ocio están aumentados en relación a los datos obtenidos a través de las encuestas de salud poblacionales correspondientes (Encuesta de Salud de Cataluña. ESCA en nuestro ámbito). Este hecho podría ser debido a que los pacientes que padecen accidentes de tráfico o laborales pueden ser atendidos inicialmente en hospitales o en mutuas laborales.

Al mismo tiempo es probable que la mayor accesibilidad y menor gravedad sean factores que determinan la atención de los accidentes en AP: un $90,2 \%$ de las consultas fueron resueltas sin necesidad de derivación, cifra parecida a la publicada en otros estudios $^{22}$ y es menor a la derivación hospitalaria realizada por los servicios de urgencias $\operatorname{AP}^{6}(20 \%)$.

Si añadimos que se observa una petición de pruebas radiológicas complementarias para el diagnóstico de $12,3 \%$, dato coincidente con estudios similares ${ }^{6,22,25}$, la polémi- ca sobre la necesidad de un mayor equipamiento en elementos de diagnóstico $(\mathrm{Rx}$, laboratorio básico) para disminuir las derivaciones y aumentar la calidad del servicio y la satisfacción del profesional y del usuario, podría trasladarse a la necesidad de un acceso rápido a estas pruebas básica para la atención urgente de AP en cualquier momento del día en el que se produce la atención, ya sea de forma ambulatoria o en un centro hospitalario.

Por último, es importante destacar que más de la mitad de los pacientes accidentados tenían menos de 20 años y un $66,6 \%$ menos de 30, predominando el sexo masculino en ambos grupos de edad. En cuanto al lugar donde se produjo el accidente, la mayoría se produjeron en el hogar (el 36,2\%) o al aire libre (el 35,2\%). Por edades, los niños entre 0-10 años se accidentaron mayoritariamente en casa y en segundo lugar en la escuela; mientras que los jóvenes entre 11-30 años se accidentaron principalmente al aire libre ${ }^{26}$. Es conocido que los accidentes infantiles en nuestro medio son la primera causa de mortalidad en el grupo de edad de 5 a 14 años y la tercera causa entre 1 y 4 $a_{n}{ }^{27}$, por lo que se han de orientar esfuerzos hacia la prevención de accidentes en este grupo de la población por todos los agentes implicados (administración, profesionales de salud, docentes y familia) ${ }^{29}$. Parece que en nuestra sociedad sí se está actuando en los accidentes graves sufridos por el grupo de edad de 14 a 24 años, pero sería interesante no olvidar la prevalencia de accidentes en los otros grupos de edad aquí mencionados.

Estos datos coinciden en parte con los referidos por otros autores ${ }^{22}$ y podrían constituir potenciales factores de riesgo para el padecimiento de accidentes. Es necesaria la realización de estudios analíticos en AP que puedan evaluar esta hipótesis, así como identificar otros potenciales factores de riesgo, para orientar de forma selectiva las medidas preventivas que permitan reducir la incidencia de accidentes en las poblaciones 
de mayor riesgo. La atención primaria puede realizar un papel importante en las intervenciones coordinadas por su atención directa y su acción comunitaria.

\section{BIBLIOGRAFÍA}

1. Oliver A, Chapí I. Estudio de la asistencia prestada en un servicio de urgencias extrahospitalario. Centro de Salud 1996; 4: 232-6.

2. Helguera F, Hormaechea E. Valoración del funcionamiento y de la patología asistida en un servicio de urgencia extrahospitalario rural. Aten Primaria 1989; 9: 292-8.

3. Ruiz T, Ronda E, Álvarez-Dardet C, Gil V, Belda J. Atención según el genero del paciente en los servicios de urgencia: ¿diferente o desigual?. Gac Sanit 1995; 9: 76-83.

4. Ibáñez F, Gutiérrez B, Olaskoaga A. Estudios de la utilización de servicios de urgencias hospitalarios por la población de un EAP: grado de adecuación. Aten Primaria 1991; 8: 764-9.

5. Blanco LE. Informe de la actividad asistencial del punto de atención continuada de Fuentes de Oñoro (Salamanca). Centro de Salud 1994; 2: 465-470.

6. Oliver A, Civera P. Estudio epidemiológico de los accidentes atendidos en un servicio de urgencias extrahospitalarias. Aten Primaria 1998; 21 : $522-6$.

7. Ministry of Health. Accident and emergency services: report of the sub-committee of the Central Health Services Council Standing Medical Advisory Committee (Chairman: Sir Harry Platt). London: HMSO; 1962.

8. Myers P. Management of minor medical problems and trauma: general practice or hospital? J Soc Med 1982; 75: 879-883.

9. Bedford HE, Jenkins SM, Shore C, Kenny PA. Use of an east end children's accident and emergency department for infants: a failure of primary health care? Quality in Health Care 1992; 1: 29-33.

10. Green J, Dale J. Primary care in accident and emergency and general practice: a comparison. Soc Sci Med 1992; 35: 987-995.
11. Driscoll PA, Vincent CA, Wilkinson M. The use of the accident and emergency department. Arch Emerg Med 1987; 4: 77-82.

12. Dale J. Primary care: the old bugbear of accident and emergency services. Br J Gen Pract 1992; 42: 90-1.

13. Cohen J. Accident and emergency services and general practice - conflict or cooperation? Fam Pract 1987; 4: 81-3.

14. Anderson NA, Gaudry P. Patients attending an accident and emergency department for primary medical care. Fam Pract 1984; 1: 79-85.

15. Bliss HA. Primary care in emergency room: high in cost and low in quality. N Engl J Med 1982; 306: 998.

16. Hansagi H, Carlsson B, Olsson M, Edhag O. Trial of method of reducing inappropriate demands on a hospital emergency department. Public Health 1987; 101: 99-105.

17. Wabschall JM. Why parents use the emergency department for non-emergency infant care. J Emerg Nursing 1983; 9: 37-40.

18. Magnusson G. The hospital emergency department as the primary source of medical care. Scand J Soc Med 1980; 8: 145-156.

19. Kljakovic M, Allan BC, Reinken J. Why skip the general practitioner and go to the accident and emergency department?. NZ Med J 1981; 96: $49-52$.

20. Área Básica de Salud número 4. Memoria 1998. Girona: Instituto Catalán de la Salud; 1999.

21. World Organization of National Colleges, Academies and Academic Associations of General Practitioners/Family Physicians. Classificacions de la WONCA per a l'atenció primària. Barcelona: Masson; 1991

22. Aarseth $\mathrm{S}$, Vatne J. Injuries in Rauma municipality in 1983. Therapeutic level costs. Tidsskr Nor Laegeforen 1991; 111: 41-4.

23. Copeman D, Skinner J, Burgin A. Occupational injury and disease among patients presenting general practitioners in a community health centre. Aust J Public Health 1992; 16: 413-8.

24. Sege R, Stigol L C, Perry C, Goldstein R, Spivak $\mathrm{H}$. Intentional injury surveillance in a primary care pediatric setting. Arch Pediatr Adolesc Med 1996, 150: 277- 283. 
25. Gofin R, Lison M, Morag C. Injuries in primary care practices. Arch Dis Child 1993; 68: 223-6.

26. Patel DR, Nelson TL. Sports injuries in adolescents. Med Clin North Am 2000 Jul; 84 (4): 983-1007.

27. Generalitat de Catalunya. Departament de Sanitat i Seguretat Social. Pla de Salut de Catlunya 1999-2001.
28. Barkin S, Fink A, Gelberg L. Predicting clinician injury prevention counseling for young children. Arch Pediatric Adolesc Med 1999 Dec; 153 (12): 1226-31.

29. Albert Cunat V, Maestro Castelblanque ME, Martínez Pérez JA, Monge Jodrá V. Factors related with accident risks in persons older than 65 years of age in the province of Guadalajara. Gac Sanit 2000 Sep-Oct; 14 (5): 346-55 